\title{
Treatment Patterns, Healthcare Resource Utilization, and Spending Among Medicaid-Enrolled Children with Chronic Idiopathic/Spontaneous Urticaria in the United States
}

\author{
Paul V. Williams · Abhishek Kavati · Dominic Pilon (D) Y Yongling Xiao • \\ Maryia Zhdanava · Maria-Magdalena Balp · Patrick Lefebvre • \\ Benjamin Ortiz $\cdot$ Vivian Hernandez-Trujillo
}

Received: November 27, 2017 / Published online: February 10, 2018

(C) The Author(s) 2018. This article is an open access publication

\section{ABSTRACT}

Introduction: Few studies have described chronic idiopathic/spontaneous urticaria (CIU/ CSU) healthcare burden in adults, while this information remains largely unknown in children. We aimed to describe treatment patterns, healthcare resource utilization (HRU), and costs in CIU/CSU pediatric patients, as well as to compare HRU and costs in CIU/CSU and CIU/ CSU-free pediatric patients.

Methods: Medicaid claims from four states (09/ 01/2013-03/31/2016) were used to identify patients less than 12 years old. The CIU/CSU

Enhanced content To view enhanced content for this article go to https://doi.org/10.6084/m9.figshare. 5821614

P. V. Williams

Northwest Asthma and Allergy Center, Everett, WA, USA

A. Kavati · B. Ortiz

Novartis Pharmaceuticals Corporation, East

Hanover, NY, USA

D. Pilon $(\bowtie) \cdot$ Y. Xiao · M. Zhdanava · P. Lefebvre Analysis Group, Inc, Montréal, QC, Canada e-mail: dominic.pilon@analysisgroup.com

M.-M. Balp

Novartis Pharma AG, Basel, Switzerland

V. Hernandez-Trujillo

Herbert Wertheim School of Medicine, Florida

International University, Miami, FL, USA cohort included patients with either at least two claims for idiopathic, other, or unspecified urticaria at least 6 weeks apart, or at least one claim for urticaria and at least one claim for angioedema at least 6 weeks apart (index date defined as the first claim). The control cohort included patients without urticaria/angioedema claims (index date randomly assigned). Patients without at least 6 months of continuous Medicaid eligibility pre- and post-index were excluded. HRU and costs were compared between propensity score-matched cohorts during the post-index follow-up.

Results: A total of $548 \mathrm{CIU} / \mathrm{CSU}$ patients (mean [SD] age 4.5 [3.3] years; $51.3 \%$ male) were matched $1: 1$ with controls. In the CIU/CSU cohort, $51.8 \%$ used non-sedating prescription $\mathrm{H}_{1}$-antihistamines, $24.3 \%$ used oral corticosteroids, and 23.5\% used other prescription $\mathrm{H}_{1}$-antihistamines; $13.5 \%$ consulted allergist/immunologists and $2.4 \%$ consulted dermatologists in the first 6 months of follow-up. Compared to controls, CIU/CSU patients had significantly more per patient per year (PPPY) inpatient (incidence rate ratio [IRR] 2.05), outpatient (IRR 2.20), and emergency department (IRR 1.64) visits (all $p$ values $<0.05)$. Moreover, CIU/CSU patients also had significantly higher PPPY healthcare costs (mean cost difference [MCD] \$1853), driven by incremental outpatient (MCD \$1286) costs (all $p$ values $<0.01$ ).

Conclusions: CIU/CSU pediatric patients had low use of non-sedating $\mathrm{H}_{1}$-antihistamines and 
high use of oral corticosteroids. Compared to CIU/CSU-free controls in the same age group, CIU/CSU pediatric patients had higher HRU and healthcare costs.

Funding: Novartis Corporation

Keywords: Children; Chronic idiopathic urticaria; Chronic spontaneous urticaria; Healthcare burden; Healthcare resource utilization; Medicaid; Pediatric; Treatment patterns

\section{INTRODUCTION}

Chronic idiopathic urticaria (CIU), also known as chronic spontaneous urticaria (CSU), is defined as the spontaneous appearance of wheals, angioedema, or both for at least 6 weeks due to known or unknown causes [1]. CIU/CSU represents $66-93 \%$ of chronic urticaria cases $[2,3]$. Prevalence of CIU/CSU in the general population is reported to be $0.5-1.0 \%$ [3]. In children, chronic urticaria is considered to be less common than in adults; however, the data on its actual prevalence is scarce $[4,5]$. Chronic urticaria has been estimated to affect $0.1-0.3 \%$ of children in the UK and $0.8 \%$ in Italy $[6,7]$. A Korean study based on patient-completed questionnaires found a prevalence of chronic urticaria in children of $1.8 \%$ [8]. Since the majority of childhood CIU/CSU cases are not seen by specialists, and are either treated by general practitioners or parents using over-thecounter (OTC) medications, obtaining valid estimates of childhood CIU/CSU prevalence and severity remains a challenge [9]. In a US health insurance claims study that used a validated algorithm to identify patients with CIU/ CSU, the prevalence of the condition was higher $(0.14 \%)$ in children compared to adolescents and adults (0.07-0.13\%) [10].

International and US guidelines on management of chronic urticaria recommend second-generation non-sedating $\mathrm{H}_{1}$ antihistamines (nsAHs) as the first-line treatment in adults, and if symptoms persist, nsAHs should be used in doses increased up to fourfold $[1,11,12]$. The same approach to treatment is suggested in children, although it is recognized that this is a weak recommendation based on clinical consensus or inadequate/low-quality evidence [1]. International guidelines recommend, as third-line therapies, adding omalizumab, leukotriene receptor antagonists (LTRAs), or cyclosporine on top of second-line treatments. A short course (up to 10 days) of oral corticosteroids (OCSs) is also suggested as third-line therapy or as an option for acute exacerbation [1]. US guidelines recommend the combinations of nsAHs with LTRAs, $\mathrm{H}_{2}$-antihistamines, cyclosporine, dapsone, or omalizumab as further lines of therapy for difficult cases in adults [12]. Although limited research suggests the use of cyclosporine in children [13], the broader use of these therapies in children is yet to be ascertained. In the USA, treatments approved specifically for children with chronic urticaria include nsAHs cetirizine [14], desloratadine [15], levocetirizine [16], and fexofenadine [17] for patients aged 6 months or more, and loratadine [18] for patients at least 2 years old.

CIU/CSU adversely affects lives of pediatric patients and their caregivers, leading to school absenteeism, reduced school performance, and more days off work [9]. Mean all-cause annual healthcare costs of CIU/CSU patients in the USA were estimated as $\$ 9142$ ( $\$ 3119$ in patients 6-11 years old), with mean urticaria-related costs comprising \$997 (\$1109 in patients 6-11 years old) [10]. Another study indicated that medication cost alone accounted for $62.5 \%$ of total annual costs of CIU/CSU patients [19]. However, little is known about the incremental economic burden of the disease in children.

To bridge the gap in knowledge about realworld treatment patterns and incremental economic burden of CIU/CSU in pediatric patients, we recently conducted a study using a large, nationally representative US commercial health insurance claims database, the results of which are submitted elsewhere for publication. The present study aimed to describe characteristics, treatment patterns, healthcare resource utilization, and costs of CIU/CSU patients (aged less than 12 years) using the Medicaid database which covers a substantial number of children in low-income families. The incremental 
healthcare resource use (HRU) and costs were assessed by comparing the outcomes in CIU/ CSU and CIU/CSU-free pediatric patients.

\section{METHODS}

\section{Data Source}

Medicaid claims from Iowa, Kansas, Missouri, and Mississippi (09/01/2013-03/31/2016) were used. The data includes medical and prescription drug claims, enrollee's plan eligibility, and demographics. All data is de-identified and in compliance with the Health Insurance Portability and Accountability Act. No institutional review board approval was required for this study.

\section{Study Design}

A retrospective cohort study design was used to evaluate baseline characteristics, treatment patterns, HRU, and costs in pediatric patients diagnosed with CIU/CSU (the CIU/CSU cohort). A retrospective matched-cohort design was used to compare HRU and costs between the CIU/ CSU cohort and CIU/CSU-free patients (the control cohort).

For both cohorts, the baseline period was defined as the 6-month period prior to the index date, and the observation period spanned from the index date until the earliest of health plan disenrollment or end of data availability.

The index date for CIU/CSU cohort was defined as the date of the first diagnosis of idiopathic, other, or unspecified urticaria or angioedema. The index date for the control cohort was randomly selected on the basis of the distribution of time between the start of the continuous eligibility period and the CIU/CSU diagnosis date in the CIU/CSU cohort.

\section{Patient Selection}

Patients in the CIU/CSU cohort were identified on the basis of the validated algorithm and had to have either at least two independent claims at least 6 weeks apart with a diagnosis of idiopathic (ICD-9-CM: 708.1x or ICD-10-CM: L50.1xx), other (ICD-9-CM: 708.8x or ICD-10CM: L50.8xx), or unspecified (ICD-9-CM: 708.9x or ICD-10-CM: L50.9xx) urticaria, or at least one claim with a diagnosis of idiopathic, other, or unspecified urticaria and at least one claim with a diagnosis of angioedema (ICD-9CM: $995.1 \mathrm{x}$ or ICD-10-CM: T78.3) at least 6 weeks apart [20]. In the control cohort, patients had no claims with a diagnosis of idiopathic, other, or unspecified urticaria or angioedema. Patients in both cohorts were required to be 11 years old or younger and have at least 6 months of continuous Medicaid eligibility before and after the index date.

\section{Study Measures}

Study measures included demographics and clinical characteristics, treatment patterns, as well as all-cause and CIU/CSU-related HRU and costs (i.e., pharmacy and medical costs) per patient per year (PPPY).

Treatment patterns were described using the following components: (1) proportions of patients using CIU/CSU-related prescription treatments, (2) proportions of patients switching CIU/CSU-related treatments and using CIU/ CSU-related treatments in combination; (3) pill burden (the ratio of the total number of pills prescribed to the number of days during a given period); and (4) types of medical specialists visited. These outcomes were described during the first 6,12 , and 18 months of follow-up among CIU/CSU patients with at least 6, 12, and 18 months of follow-up, respectively.

HRU and costs were reported by type of service: inpatient (IP), emergency department (ED), and outpatient (OP; broken down into urgent care facility, home care, office, ambulatory surgery center, and other OP). All-cause HRU and costs were assessed during the observation period for both cohorts. CIU/CSU-related HRU and costs were defined using diagnosis codes for idiopathic, other, or unspecified urticaria, and were described for the CIU/CSU cohort only. All costs were inflation-adjusted to 2016 dollars based on the US Consumer Price Index. 


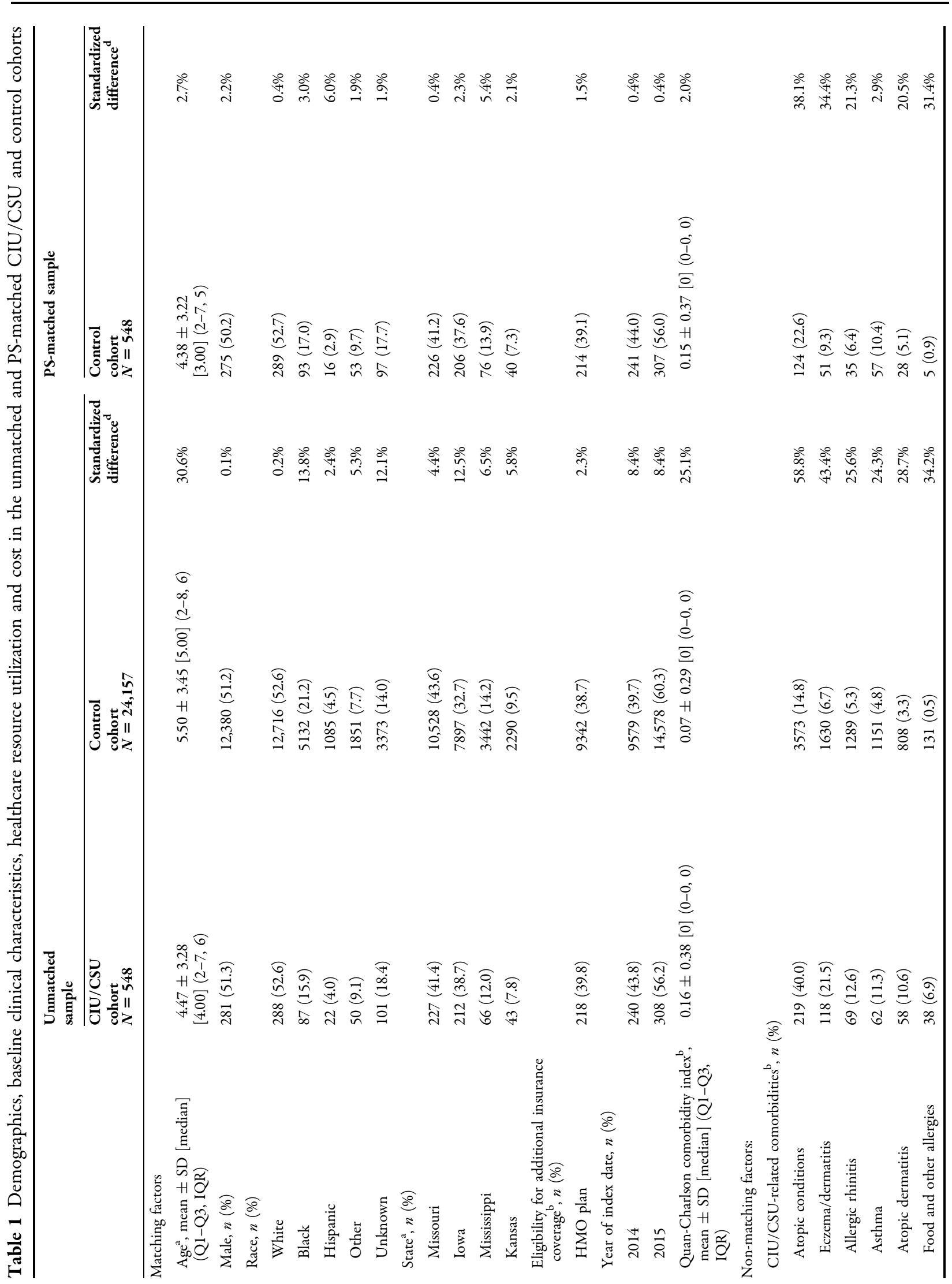




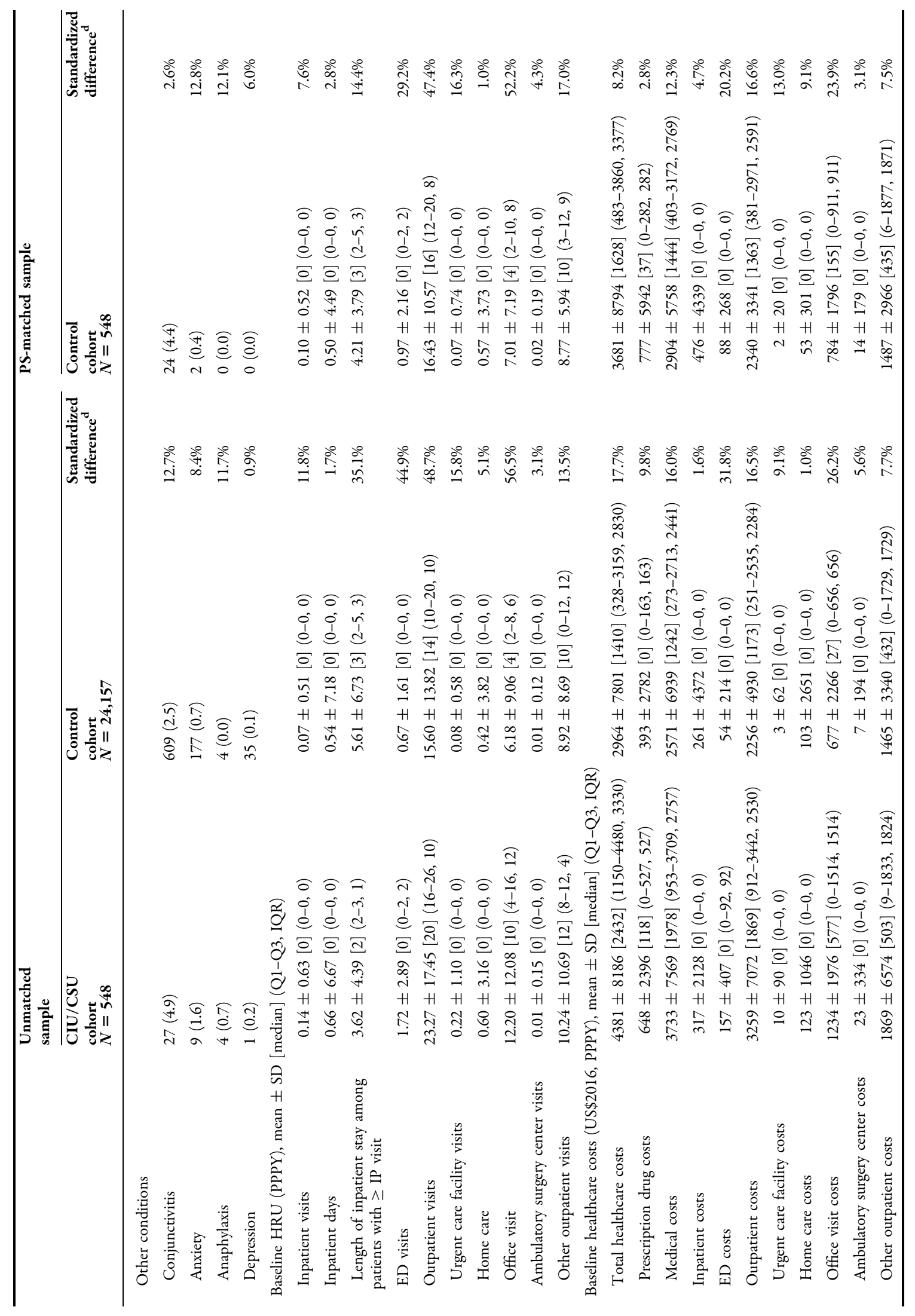




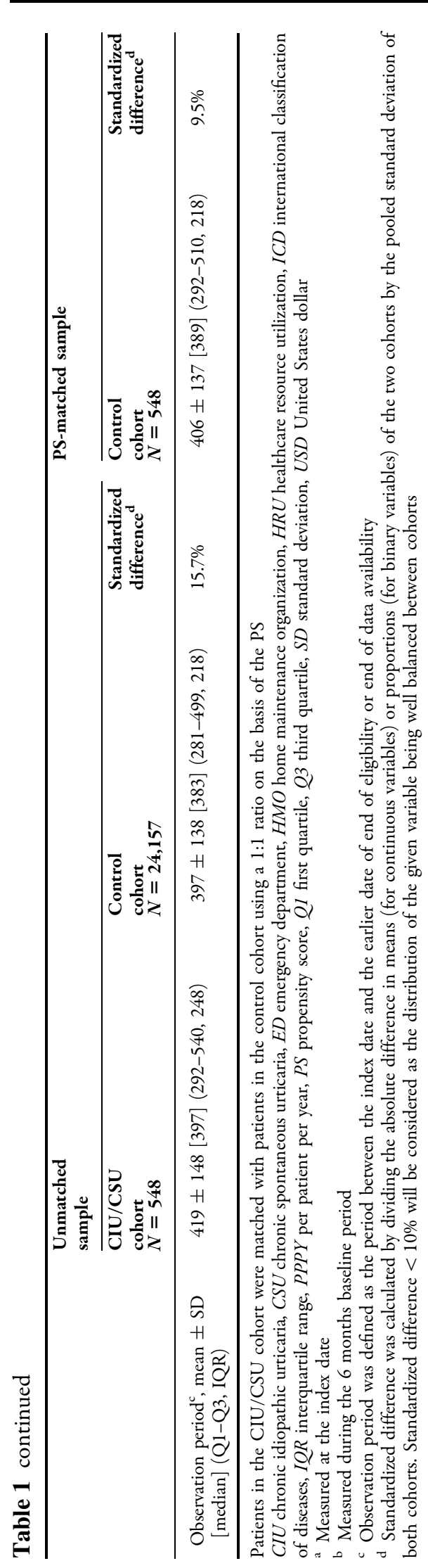

\section{Statistical Analysis}

Mean, standard deviation (SD), first and third quartile, interquartile range (IQR), and median were used to describe continuous variables, and frequencies and percentages were used to describe categorical variables.

Propensity score (PS) matching was used to adjust for the differences in baseline characteristics between the CIU/CSU and control cohorts. PS, defined as the probability of being in the CIU/CSU cohort, was estimated using a multivariate logistic regression model, including age, sex, race, state, presence of Home Maintenance Organization (HMO) insurance plan, year of the index date, and the QuanCharlson comorbidity index (Quan-CCI). Patients in the CIU/CSU cohort were matched in a 1:1 ratio with control patients on the basis of PS. A standardized difference calculated by dividing the absolute difference in means (for continuous variables) or proportions (for categorical variables) of two cohorts by the pooled SD of both cohorts was used to assess the balance of baseline characteristics between cohorts before and after matching (less than 10\% indicated sufficient balance) [21].

All-cause HRU and costs were compared between the PS-matched CIU/CSU and control cohorts using univariate Poisson and linear regression models. For HRU, the 95\% confidence intervals (CIs) and $p$ values for incidence rate ratios (IRRs) were calculated using the robust variance estimator to account for the correlation in PS-matched data. For costs, the 95\% CIs and $p$ values for mean cost differences (MCDs) were based on a bootstrap approach (500 resamples) to account for non-normal distribution of cost data, and used robust variance estimator to account for the correlation in PS-matched data. All analyses were performed using SAS version 9.4 (SAS Institute, NC, US).

\section{RESULTS}

\section{Demographics and Clinical Characteristics}

Figure 1 summarizes the study cohort selection. A total of $2270 \mathrm{CIU} / \mathrm{CSU}$ patients were 


\section{CIU/CSU cohort}

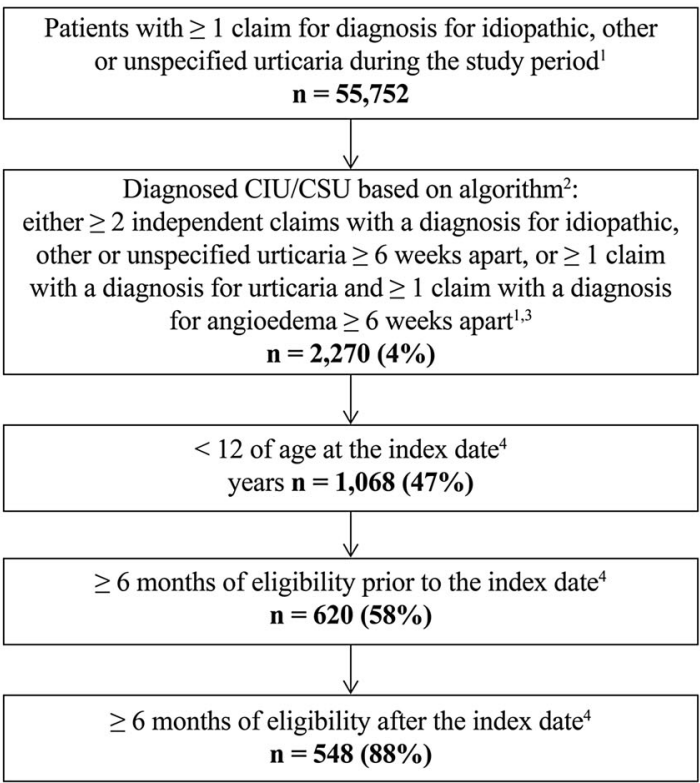

Fig. 1 Cohort selection flowcharts. CIU chronic idiopathic urticaria, CSU chronic spontaneous urticaria. ${ }^{1}$ Idiopathic urticaria was defined on the basis of ICD-9CM diagnostic code 708.1x and ICD-10-CM diagnostic code L50.1xx; other urticaria was defined on the basis of ICD-9-CM diagnostic code 708.8x and ICD-10-CM diagnostic code L50.8xx; unspecified urticaria was defined on the basis of ICD-9-CM diagnostic code 708.9x and ICD-10-CM diagnostic code L50.9xx. ${ }^{2}$ Claims algorithm for identifying patients with CIU/CSU was validated by using those reported by Cherepanov et al. [20]. ${ }^{3} \mathrm{An}$ gioedema was defined on the basis of ICD-9-CM diagnostic code 995.1x and ICD-10-CM diagnostic code

identified, and 548 met the remaining inclusion criteria. The mean (SD) age of CIU/CSU patients was 4.5 (3.3) years, patients were predominantly male $(51.3 \%)$, white $(52.6 \%)$, residing in Missouri $(41.4 \%)$ or Iowa $(38.7 \%), 39.8 \%$ were additionally covered by an HMO plan (Table 1 ). Atopic conditions were observed in $40.0 \%$ of CIU/CSU patients. The mean (SD) Quan-CCI was 0.16 (0.38). All $548 \mathrm{CIU} / \mathrm{CSU}$ patients were matched 1:1 with patients in the control cohort. After matching, demographics and Quan-CCI were comparable in both cohorts, and the duration of follow-up period was similar.
Control cohort

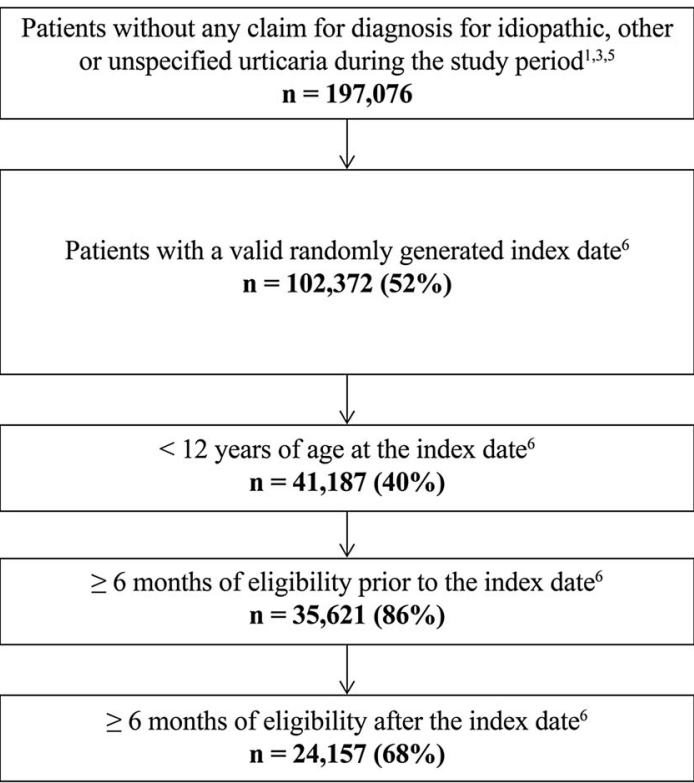

T78.3. ${ }^{4}$ Index date for the CIU/CSU cohort was defined as the date of first claim for idiopathic, other, or unspecified urticaria or angioedema during the study period. ${ }^{5}$ In each state, a 5\%-random sample of patients without diagnoses of interest was selected. ${ }^{6}$ Index date for the control cohort was randomly selected on the basis of the distribution of time between the start of the continuous eligibility period and the index date observed in the CIU/CSU cohort to mimic the index date of CIU/ CSU patients. Note that a patient with a randomly generated index date that was beyond the end of his/her enrollment period was excluded

\section{Treatment Patterns in CIU/CSU Cohort}

An increase in use of CIU/CSU-related medications from the baseline period was observed in the CIU/CSU cohort during the 6 months of follow-up. Specifically, $21.0 \%$ vs $51.8 \%$ used prescription nsAHs (i.e., cetirizine, desloratadine, fexofenadine, levocetirizine, and loratadine), $9.3 \%$ versus $24.3 \%$ used OCSs, $8.9 \%$ vs $23.5 \%$ used other $\mathrm{H}_{1}$-antihistamines (i.e., all other $\mathrm{H}_{1}$-antihistamines not listed as nsAHs), and $7.7 \%$ vs $15.7 \%$ used LTRAs (Table 2 ). The proportion of patients using CIU/CSU therapies in combination was small, only $15.7 \%$ of patients switched CIU/CSU therapies, and mean 
(SD) daily-prescription pill burden was 3.0 (3.5) during the 6 months of follow-up. Proportions of patients seen by allergists or immunologists $(0.9 \%$ vs $13.5 \%)$ or dermatologists $(1.6 \%$ vs $2.4 \%$ ) increased from the baseline period compared to the 6 months of follow-up, but remained relatively low.

\section{HRU and Costs}

Comparison of all-cause HRU between PS-matched cohorts revealed a significantly higher rate of visits PPPY in the CIU/CSU cohort compared to the control cohort (Table 3). Specifically, patients in the CIU/CSU cohort had about twice as many IP visits (IRR 2.05, $p=0.031$ ) and ED visits (IRR 2.20, $p<0.001$ ), and $64 \%$ more OP visits (IRR $1.64, p<0.001$ ). In terms of subcategories of OP visits, patients in the CIU/CSU cohort had about twice as many office (IRR 2.36, $p<0.001$ ) and $21 \%$ more other OP (IRR 1.21, $p<0.001)$ visits.

In the CIU/CSU cohort, CIU/CSU-related IP visits comprised $26.7 \%$ of all-cause IP visits, CIU/CSU-related ED visits comprised $29.9 \%$ of all-cause ED visits, and CIU/CSU-related OP visits comprised $10.3 \%$ of all-cause OP visits.

Consistently with higher HRU, patients in the CIU/CSU cohort had higher healthcare costs PPPY compared to patients in the control cohort (Table 4). Specifically, all-cause pharmacy and medical costs were $\$ 4149$ PPPY in the CIU/CSU cohort compared to \$2295 PPPY in the control cohort (MCD \$1853, $p<0.001$ ). Incremental OP costs (MDC \$1286, $p<0.001$ ) represented the majority ( $82.2 \%)$ of incremental medical costs, and incremental office visits costs (MCD \$851, $p<0.001$ ) represented the majority $(66.2 \%)$ of incremental OP costs. All subcategories of OP costs (with the exception of ambulatory surgery center costs) as well as ED costs were significantly higher in the CIU/CSU cohort compared to the control cohort; however, the numerical differences were relatively small. Pharmacy costs were numerically higher in the CIU/CSU cohort, but the difference was non-significant (MCD \$288, $p=0.196$ ).

In the CIU/CSU cohort, CIU/CSU-related total healthcare costs represented $9.9 \%$ of all- cause costs and comprised $22.1 \%$ of the incremental total healthcare costs of CIU/CSU patients compared to control patients.

\section{DISCUSSION}

In this study, data on a large sample of US children covered by Medicaid was analyzed to address a knowledge gap about real-world treatment patterns and economic burden of pediatric patients diagnosed with CIU/CSU.

In terms of treatment patterns, this study revealed that after the CIU/CSU diagnosis, nsAHs (predominantly cetirizine) were the most commonly prescribed treatments, and that a considerable proportion of pediatric CIU/CSU patients also received OCSs, other $\mathrm{H}_{1}$-antihistamines, and LTRAs (montelukast). The use of nsAHs in this study (51.8\%) was much higher than in a commercially insured CIU/CSU pediatric population $(8.0 \%$ [22]), which may explain the higher pill burden and higher pharmacy costs seen in this study. The higher use of other $\mathrm{H}_{1}$-antihistamines in Medicaid is not surprising, as Medicaid covers oral histamines in many states when prescribed, whereas many commercial insurance companies do not cover $\mathrm{H}_{1}$ antihistamines. The overall use of antihistamines was about $75 \%$, and considering the widespread use of diphenhydramine in the pediatric population, which is readily available OTC and not by prescription, would suggest that the use of $\mathrm{H}_{1}$-antihistamines was appropriate. However, considering the recommendations of international guidelines, the use of nsAHs was low. Several prior studies (although mostly involving adult patients) have reported OCSs and LTRAs as some of the most frequently used treatments in CIU/CSU [10, 19, 23]. Proportions of patients using OCS and LTRAs in this study may provide a rough estimate of pediatric CIU/CSU patients uncontrolled on $\mathrm{H}_{1}$-antihistamines. The use of LTRAs is reported to be well tolerated and have low side effect profiles [24]. However, pediatric patients treated with OCSs are shown to face an increased risk of side effects such as fractures, impaired growth, vomiting, depression etc. which could results in higher total healthcare costs [25]. Similarly, the use of first-generation 


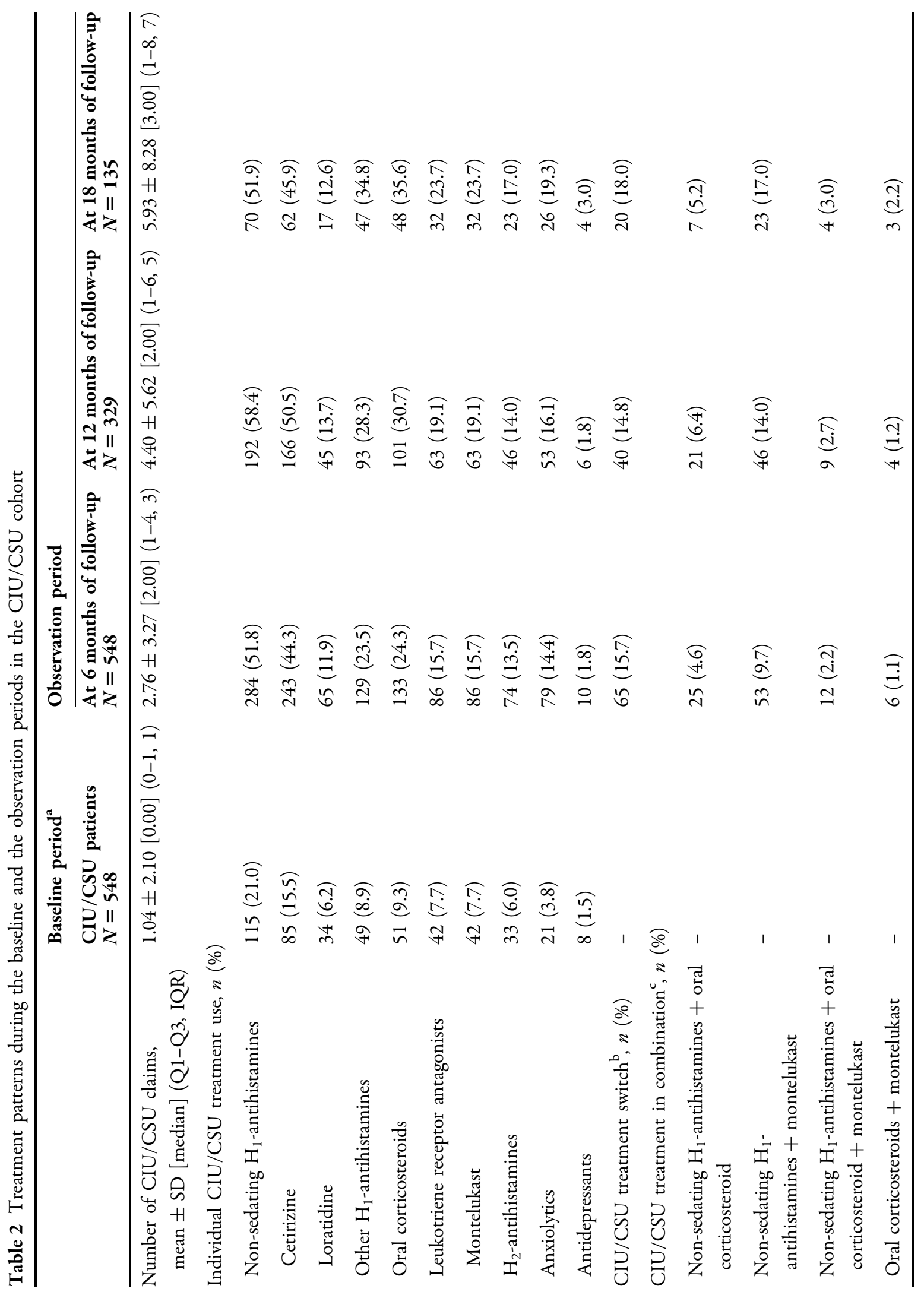




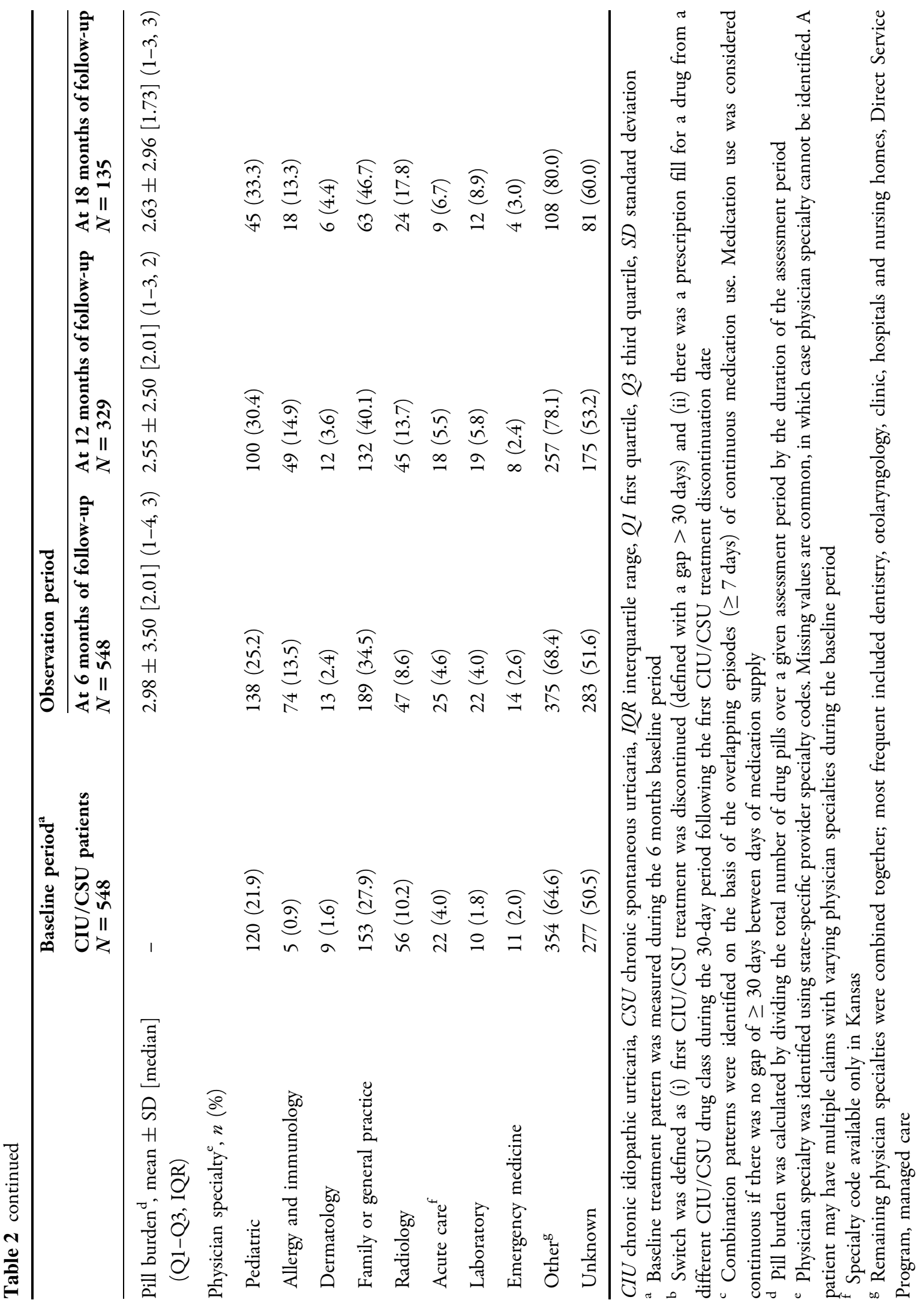




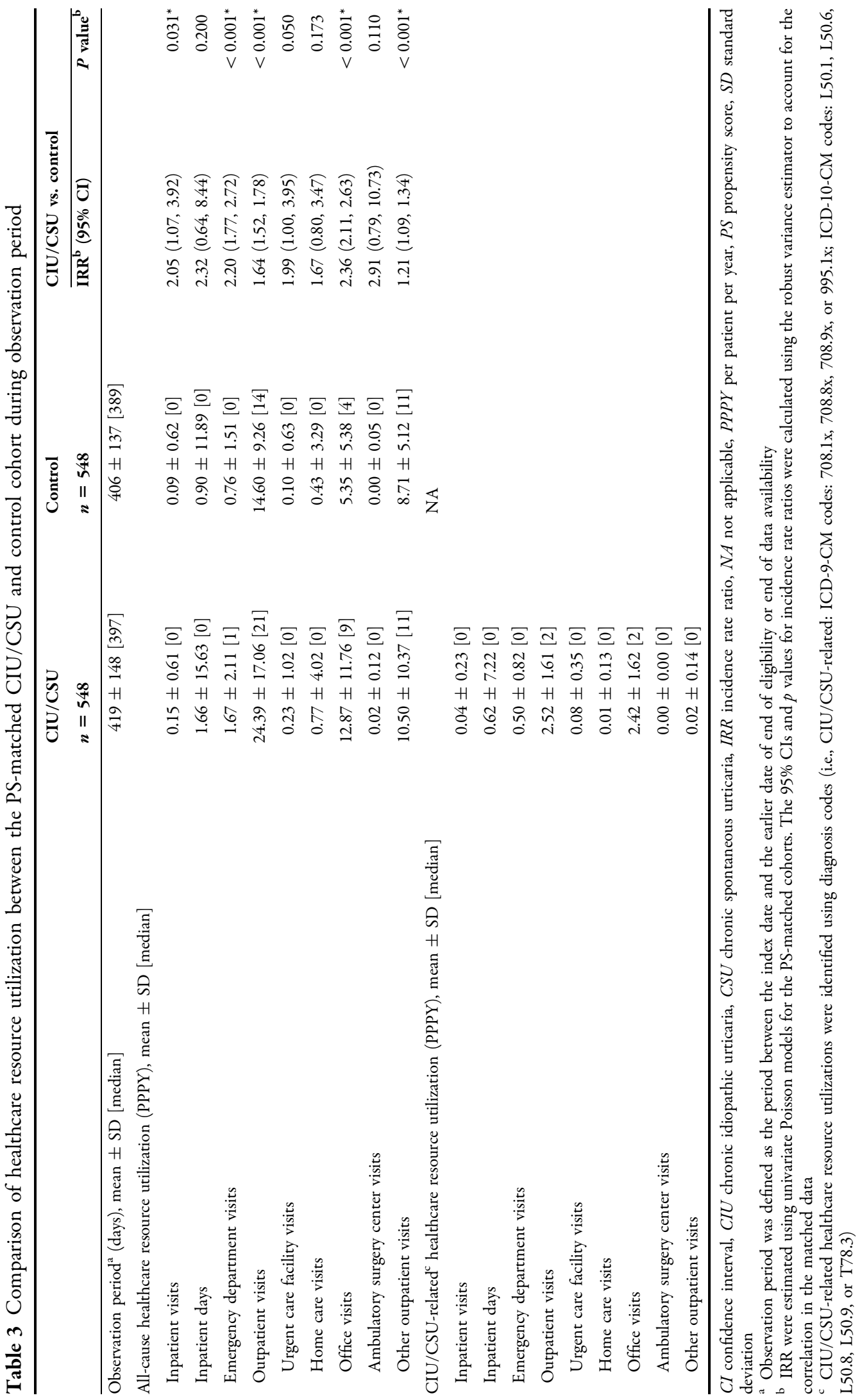




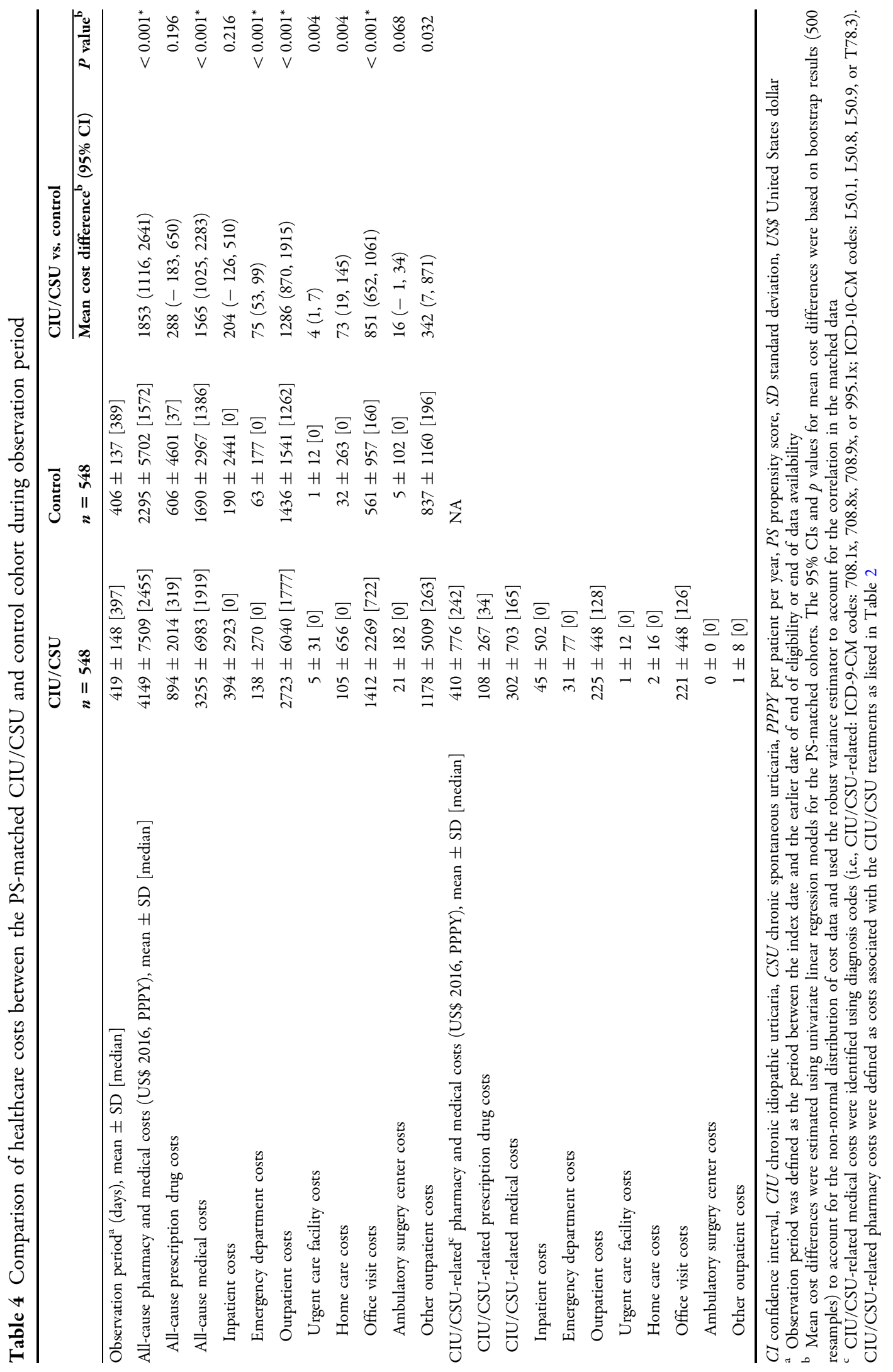


$\mathrm{H}_{1}$-antihistamines should be avoided in pediatric patients because of their potential to cause adverse events even when administered in licensed doses [9]. In this study, the low proportion of patients with a visit to an allergists/ immunologist or dermatologist might partially reflect the fact that the data on physician specialty was not well populated in Medicaid. At the same time, the low proportion of patients with visits to specialists seems to be consistent with the understanding that the majority of pediatric patients with CIU/CSU are treated either by general physicians and pediatricians or by parents with OTC medications [9].

The literature on economic burden of CIU/ CSU in pediatric patients is limited, which complicates an extensive comparison of results obtained in this study to prior research. Nevertheless, the number of all-cause office visits PPPY (12.9) was consistent with the number reported for US commercially insured patients under 5 years of age (12.0) and aged between 6 and 11 years old (10.5) [10]. Mean annual healthcare costs of pediatric patients were also relatively consistent, but slightly higher, between the two studies (\$4149 in the current study versus $\$ 2676$ in patients under 5 years of age and $\$ 3119$ in patients aged 6 and 11 years old in the prior study on US commercially insured patients) [10]. Moreover, the number of all-cause office visits PPPY observed in this study appeared to be similar to that in commercially insured US adults with CIU/CSU (14.5 [23], 15.1 [10]), but all-cause healthcare costs PPPY represented approximately a half of costs reported in adults (\$9142) [10]. The incremental total healthcare costs in this study constituted approximately \$2000 PPPY, and were mostly driven by incremental OP costs. This finding is consistent with the observation that CIU/CSU is essentially an OP managed condition $[10,19]$. Total healthcare cost directly related to CIU/ CSU comprised \$410 PPPY (approximately 10\% of the all-cause costs) suggesting that a significant proportion of the CIU/CSU burden can be explained by comorbidities associated with CIU/CSU. All-cause and disease-related total healthcare costs in this study were comparable to those reported in asthma (\$3076 and \$507
PPPY, respectively) among US school-aged children 6 to 17 years old [26].

This study was subject to several limitations. First, the Medicaid data was obtained from only four states during a limited study period and may not be representative of the overall US population. Second, only prescription antihistamines are captured in pharmacy claims. Since antihistamines could be obtained as OTC, the current study likely underreports their use. Third, prescription fills captured in pharmacy claims data do not guarantee whether medications were actually taken as prescribed. This may overestimate the actual medication consumption. Fourth, the reliability of the algorithm to identify CIU/CSU among pediatric patients is yet to be confirmed. Fifth, claims data have inherent limitations including potential miscoding and/or missing data. However, these limitations are unlikely to have a systematic effect on results and claims data remain a valuable source of information on realworld patients. Finally, the results of this study may be subject to residual confounding.

\section{CONCLUSIONS}

The results of this study suggested that CIU/ CSU patients aged less than 12 years had an increased medication burden following a CIU/ CSU diagnosis. CIU/CSU pediatric patients had low nsAHs use and high OCSs use. Compared to patients in the same age group without CIU/ CSU, patients with CIU/CSU had higher HRU and costs. The incremental cost burden constituted about $\$ 2000$ per patient per year, driven primarily by OP costs and visits. These findings underscore the importance of prompt diagnosis and use of appropriate therapies in this population.

\section{ACKNOWLEDGMENTS}

Funding. This study was funded by Novartis Pharmaceuticals Corporation. Article processing charges were funded by Novartis Pharmaceuticals Corporation. All authors had full access to 
all of the data in this study and take complete responsibility for the integrity of the data and accuracy of the data analysis.

Authorship. All named authors meet the International Committee of Medical Journal Editors (ICMJE) criteria for authorship for this manuscript, take responsibility for the integrity of the work as a whole, and have given final approval for the version to be published.

Medical Writing Assistance. Medical writing assistance was provided by Raju Gautam, PhD, an employee of Novartis Healthcare Pvt. Ltd., Hyderabad, India.

Disclosures. P. Lefebvre is an employee of Analysis Group. D. Pilon is an employee of Analysis Group. Y. Xiao is an employee of Analysis Group. M. Zhdanava is an employee of Analysis Group. Analysis Group is a consulting company that has received research grants from Novartis Pharmaceuticals Corporation to conduct this study. A. Kavati is an employee of Novartis Pharmaceuticals Corporation. B. Ortiz is an employee of Novartis Pharmaceuticals Corporation. M.M. Balp is an employee of Novartis Pharma AG. P.V. Williams received consulting fees from Novartis Pharmaceuticals Corporation. V. Hernandez-Trujillo received consulting fees from Novartis Pharmaceuticals Corporation. V. Hernandez-Trujillo also served as a consultant to Bayer.

Compliance with Ethics Guidelines. This retrospective study used de-identified data that complied with the Health Insurance Portability and Accountability Act. No institutional review board approval was required for this study.

Data Availability. The datasets generated during and/or analyzed during the current study are available from the corresponding author on reasonable request.

Open Access. This article is distributed under the terms of the Creative Commons Attribution-NonCommercial 4.0 International License (http://creativecommons.org/licenses/ by-nc/4.0/), which permits any noncommercial use, distribution, and reproduction in any medium, provided you give appropriate credit to the original author(s) and the source, provide a link to the Creative Commons license, and indicate if changes were made.

\section{REFERENCES}

1. Zuberbier $\mathrm{T}$, Aberer $\mathrm{W}$, Asero R, et al. The EAACI/ GA(2) LEN/EDF/WAO Guideline for the definition, classification, diagnosis, and management of urticaria: the 2013 revision and update. Allergy. 2014;69(7):868-87.

2. Goldstein S, Weinberg JM. Recurrent and persistent urticaria: is it chronic idiopathic urticaria? Narrative review on diagnosis and management. J Dermatol Nurses Assoc. 2016;8(4):250-60.

3. Maurer $\mathrm{M}$, Weller $\mathrm{K}$, Bindslev-Jensen $\mathrm{C}$, et al. Unmet clinical needs in chronic spontaneous urticaria. A GA(2)LEN task force report. Allergy. 2011;66(3):317-30.

4. Poddighe D, De Amici M, Marseglia GL. Spontaneous (autoimmune) chronic urticaria in children: current evidences, diagnostic pitfalls and therapeutic management. Recent Pat Inflamm Allergy Drug Discov. 2016;10(1):34-9.

5. Shin M, Lee S. Prevalence and causes of childhood urticaria. Allergy Asthma Immunol Res. 2017;9(3):189-90.

6. Cantarutti A, Dona D, Visentin F, et al. Epidemiology of frequently occurring skin diseases in Italian children from 2006 to 2012: a retrospective, population-based study. Pediatr Dermatol. 2015;32(5):668-78.

7. Khakoo G, Sofianou-Katsoulis A, Perkin MR, Lack G. Clinical features and natural history of physical urticaria in children. Pediatr Allergy Immunol. 2008;19(4):363-6.

8. Lee SJ, Ha EK, Jee HM, et al. Prevalence and risk factors of urticaria with a focus on chronic urticaria in children. Allergy Asthma Immunol Res. 2017;9(3):212-9.

9. Church MK, Weller K, Stock P, Maurer M. Chronic spontaneous urticaria in children: itching for insight. Pediatr Allergy Immunol. 2011;22(1 Pt 1):1-8. 
10. Broder MS, Raimundo K, Antonova E, Chang E. Resource use and costs in an insured population of patients with chronic idiopathic/spontaneous urticaria. Am J Clin Dermatol. 2015;16(4):313-21.

11. Beck LA, Bernstein JA, Maurer M. A review of international recommendations for the diagnosis and management of chronic urticaria. Acta Derm Venereol. 2017;97(2):149-58.

12. Bernstein JA, Lang DM, Khan DA, et al. The diagnosis and management of acute and chronic urticaria: 2014 update. J Allergy Clin Immunol. 2014;133(5):1270-7.

13. Neverman L, Weinberger M. Treatment of chronic urticaria in children with antihistamines and cyclosporine. J Allergy Clin Immunol Pract. 2014;2(4):434-8.

14. U.S. Food and Drug Administration (FDA). ZYRTEC $^{\circledR}$ (cetirizine hydrochloride) Tablets and Syrup, 2003.

15. U.S. Food and Drug Administration (FDA). CLARINEX (desloratadine) full prescribing information, 2014.

16. U.S. Food and Drug Administration (FDA). XYZAL (levoceterizine dihydrochloride) full prescribing information, 2010.

17. U.S. Food and Drug Administration (FDA). ALLEGRA (fexofenadine hydrochloride) full prescribing information, 2007.

18. U.S. Food and Drug Administration (FDA). CLARITIN (brand of loratadine) product information, 2000 .

19. Delong LK, Culler SD, Saini SS, Beck LA, Chen SC. Annual direct and indirect health care costs of chronic idiopathic urticaria: a cost analysis of 50 nonimmunosuppressed patients. Arch Dermatol. 2008;144(1):35-9.

20. Cherepanov D, Raimundo K, Chang E, et al. Validation of an ICD-9-based claims algorithm for identifying patients with chronic idiopathic/spontaneous urticaria. Ann Allergy Asthma Immunol. 2015;114(5):393-8.

21. Austin P. Using the standardized difference to compare the prevalence of a binary variable between two groups in observational research. Commun Stat - Simul Comput. 2009;38(6):1228.

22. Williams P, Lefebvre P, Pilon D, et al. Real-world treatment patterns in pediatric patients diagnosed with chronic idiopathic or spontaneous urticaria in the United States. Poster presented at the 26th European Academy of Dermatology and Venereology (EADV) Congress, September 13-17, 2017, Geneva, Switzerland. 2017.

23. Zazzali JL, Broder MS, Chang E, Chiu MW, Hogan DJ. Cost, utilization, and patterns of medication use associated with chronic idiopathic urticaria. Ann Allergy Asthma Immunol. 2012;108(2):98-102.

24. de Silva NL, Damayanthi H, Rajapakse AC, Rodrigo C, Rajapakse S. Leukotriene receptor antagonists for chronic urticaria: a systematic review. Allergy Asthma Clin Immunol. 2014;10(1):24.

25. Manson SC, Brown RE, Cerulli A, Vidaurre CF. The cumulative burden of oral corticosteroid side effects and the economic implications of steroid use. Respir Med. 2009;103(7):975-94.

26. Sullivan PW, Ghushchyan V, Navaratnam P, et al. The national cost of asthma among school-aged children in the United States. Ann Allergy Asthma Immunol. 2017;119:246-52. 